\title{
Axillary Lymph Node Metastasis of Colon Cancer-Case Report and Literature Review
}

\author{
Helena Devesa, Luísa Pereira, Álvaro Gonçalves, Telma Brito, Teresa Almeida, \\ Rui Torres, Alberto Midões \\ Serviço de Cirurgia Geral, Unidade Local de Saúde do Alto Minho, Viana do Castelo, Portugal \\ Email: helenadevesa@gmail.com
}

Received 9 November 2014; revised 1 December 2014; accepted 20 December 2014

Copyright (C) 2014 by authors and Scientific Research Publishing Inc.

This work is licensed under the Creative Commons Attribution International License (CC BY). http://creativecommons.org/licenses/by/4.0/

(c) (i) Open Access

\begin{abstract}
The most common metastatic sites of colon cancer are local lymph nodes, liver and lungs. Lymph node metastasis at distant sites is rare. In this article, we describe the case of a patient with ascending colon cancer with a metastasis in an axillary lymph node. The clinical presentation of this tumor was with an intestinal obstruction, and an emergency resection surgery was undertaken. The patient died at the third post-operative month, with lymph node and hepatic metastasis, and implants in the abdominal fat and in the mesentery, near the ileocolic anastomosis.
\end{abstract}

\section{Keywords}

\section{Colon Cancer, Axillary Metastasis, Unusual Metastasis}

\section{Introduction}

The most common metastatic sites of right colon cancer are local lymph nodes and liver. Other common metastatic sites are lung, peritoneum and ovary. Disease dissemination to lymph nodes other than the local ones is rare, with only four cases reported, as far as we know, of disease recurrence in axillary lymph nodes in patients with primary cancer of the colon [1]-[4].

In this article, we describe the case of a woman with a right colon cancer that had a metastasis in an axillary lymph node at disease presentation time. This cancer presented as an intestinal obstruction with perforation, needing emergent surgery, and a right hemicolectomy was performed. At the time of death, the patient had lymph node and hepatic metastasis, and implants in the abdominal fat and in the mesentery, near the ileocolic anastomosis.

\section{Case Report}

An 82-year-old woman presented in the Emergency Department with an abdominal pain, diarrhea and fever,

How to cite this paper: Devesa, H., Pereira, L., Gonçalves, Á., Brito, T., Almeida, T., Torres, R. and Midões, A. (2014) Axillary Lymph Node Metastasis of Colon Cancer-Case Report and Literature Review. Case Reports in Clinical Medicine, 3, 669-673. http://dx.doi.org/10.4236/crcm.2014.312141 
with 24 hours of evolution.

At clinical examination, she presented pale, with fever $\left(38.7^{\circ} \mathrm{C}\right)$ and tachycardia, without hypotension. Her abdomen was soft, painful at the right hypochondrium and right flank palpation, without tenderness nor guardness.

Her blood test showed anaemia (haemoglobin of $6.8 \mathrm{~g} / \mathrm{dl}$ ) and elevated C reactive protein $(27.12 \mathrm{mg} / \mathrm{dl})$, with no leukocytosis.

\subsection{Imaging}

The patient had an abdominopelvic CT scan that showed a thickening of the right colon wall causing a serious stenosis of the colon lumen, with moderate dilatation of the cecum. This lesion showed no signs of duodenum invasion nor other adjacent structures invasion. The tumor was perforated, showing a small volume pneumoperitoneum in the scan. There were no liver metastasis, nor alterations in the spleen, pancreas, kidneys nor adrenal glands. There were no abdominopelvic enlarged lymph nodes (Figure 1(a) and Figure 1(b)).

\subsection{Treatment}

The patient underwent urgent surgery. The surgery findings were a right colon tumor, adherent to the posterior abdominal wall, with a small amount of pus in right parietocolic gutter. There were no liver metastasis nor peritoneal carcinomatosis. A right hemicolectomy was performed, with a side-to-side mechanical ileocolic anastomosis.

The histologic diagnosis of the surgical specimen was adenocarcinoma of the colon, with infiltration of the subserosa and perforation of the visceral peritoneum, and metastasis in 7 of the 17 pericolic lymph nodes isolated, with extra-nodal extension (stage pT4aN2b). The patient was not tested for Kras mutation.

Staging of the tumor was completed with a thorax CT scan that identified an enlarged lymph node in the left axilla with $15 \mathrm{~mm}$ in diameter. There were no lung metastasis nor mediastinicenlarged lymph nodes (Figure 2(a) and Figure 2(b)).

A biopsy of the axillary lymph node was undertaken, and its histologic evaluation showed structures of adenocarcinoma involving lymphoid parenchyma, with expression of the marker CK 20, compatible with a colon cancer metastasis (Figure 3(a) and Figure 3(b)).

The post-operative period was complicated with surgical site infection and an anastomotic leak with abscess formation, which was managed conservatively.

One month and a half after the surgery, the patient underwent a PET scan that showed malignant infiltration of the mesentery near the ileocolic anastomosis, abdominal lymph node metastasis, liver metastasis and an abdominal fat implant.

She was evaluated by a multidisciplinary team that decided for palliative chemotherapy.

The patient was discharged at $58^{\text {th }}$ postoperative day, with no complaints and with regular bowel movements. She died at the $3^{\text {rd }}$ postoperative month of disease progression.

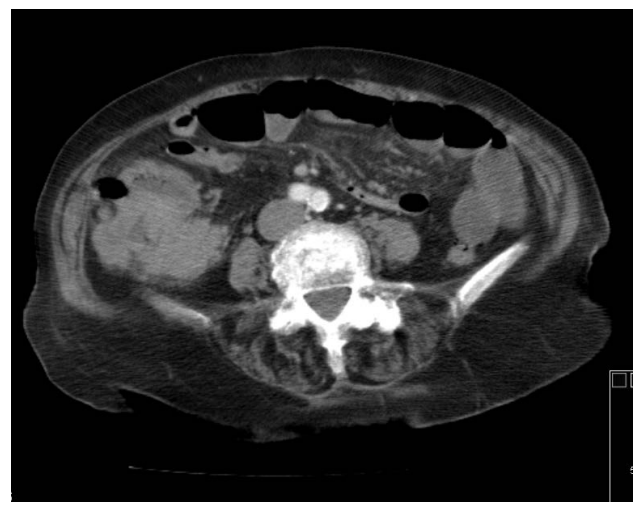

(a)

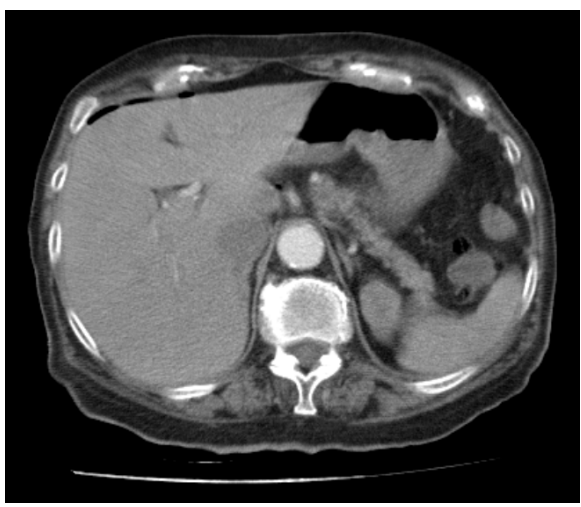

(b)

Figure 1. (a) Abdominal CT scan showing a right colon tumor with perforation; (b) Abdominal CT scan showing a small volume pneumoperitoneum. 


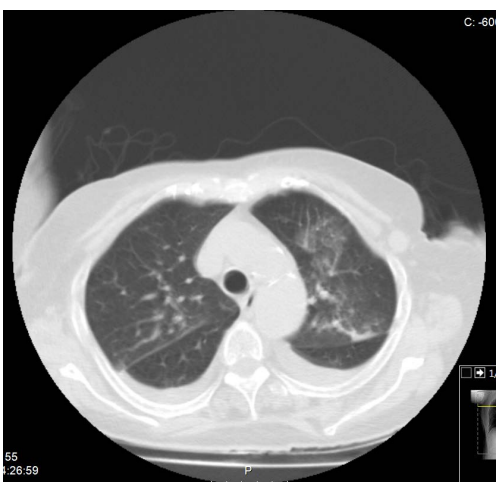

(a)

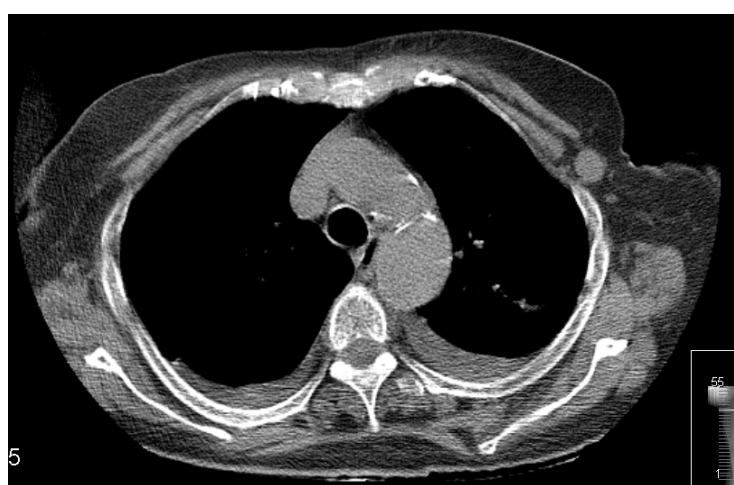

(b)

Figure 2. (a) Thorax CT scan showing an enlarged lymph node in the left axilla (arrow); (b) Thorax CT scan showing an enlarged lymph node in the left axilla (arrow).

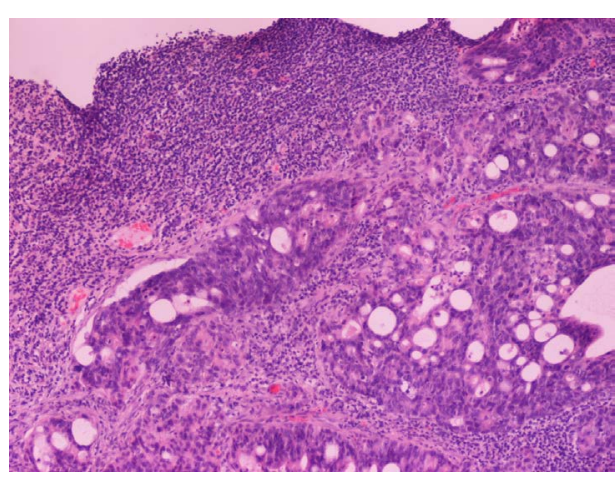

(a)

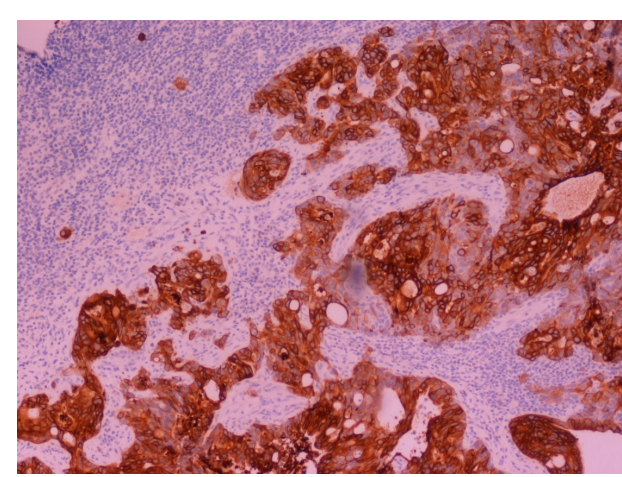

(b)

Figure 3. (a) Presence of neoplastic structures with glandular and cribiform pattern in the middle of lymphoid parenchyma, with epithelial proliferation and atypia, compatible with metastatic adenocarcinoma; (b) Expression of the immunohistochemical marker CK20 in the tumoral cells, which in the clinical context is compatible with a colorectal cancer.

\section{Discussion}

Metastatic sites of colon cancer are quite predictable, occurring initially by lymphatic spread and afterwards by haematogenous spread [3]. Lymph node metastasis is one of the most important prognostic factors in patients with colorectal cancer, and many studies have indicated that the location and number of metastatic nodes affect prognosis [5]-[9]. However, information regarding the mechanism or process of the metastatic spread of tumor cells to distant regional lymph nodes in patients with colorectal carcinoma is sparse at present. There are studies demonstrating that the presence of extracapsular invasion at metastatic lymph nodes is significantly related to prognosis not only in colorectal carcinoma but also in various other types of carcinoma [5] [6]. The presence of extracapsular invasion in positive lymph nodes is significantly related to the nodal spread of the tumor cells in colorectal cancer patients and may partially explain the mechanism of dissemination to distant regional lymph nodes. Tumor cells invade the lymphatic vessels, which enables the tumor cells to penetrate into the lymphatic system [5].

The usual pattern of local lymph node metastasis follows the vascular distribution of the mesocolon. The tumors of the cecum spread through ileocolic lymph nodes, while ascending colon tumors and proximal transverse colon tumors drain in the lymph nodes of the right colic and middle colic arteries. These nodes drain in the lymph nodes that accompany the superior mesenteric artery. Descending colon tumors spread to the lymph nodes of the left colic artery, while the tumors of the sigmoid colon drain in the sigmoid arteries lymph nodes. These nodes drain in the lymph nodes that accompany the inferior mesenteric artery. The superior and inferior mesenteric arteries nodes belong to the pre-aortic lymph nodes. The lymphatic drainage of all colorectal tumors ends in the cisterna chyli that drains into the thoracic duct [10]. 
Most common sites of colorectal cancer metastasis are local lymph nodes (50\% - 70\%) and liver (35\% - 50\%). Other common sites are lung (21\%), peritoneum (15\%) and ovaries (13.1\%). Rare distant metastatic sites of colonic carcinoma are central nervous system (8.3\%), bone (8.7\%), kidney (6.6\%), testis, penis, uterus and oral cavity. Very rare metastatic sites like adrenal gland (4.3\%), hilar lymph nodes, skin and muscles have been described, and occasional cases of metastasis to pancreas, maxillary sinus, thyroid and knee synovial have been reported [3]. Therefore, although local lymph nodes, liver and lungs are the most common initial sites of colonic carcinoma metastasis, disseminated metastasis that spare these organs might occur [3].

In many cases, the spread pathway of rare metastasis is not well defined. If tumor cells invade blood vessels, they may originate a distant cutaneous metastasis, while if they invade lymphatic vessels, the most common result will be a late local recurrence [3] [11].

Cutaneous metastasis or metastatic lymph nodes near the skin surface, like Virchow lymph node in gastric cancer, or inguinal lymph nodes in rectal cancer, occur mainly in extensive metastatic disease and are associated witha poor prognosis [1] [11]. However, colorectal cancer metastasis to distant non-regional lymph nodes other than the inguinal ones is rare, namely to the axillary nodes [1].

In a study published in 2006 in the American Cancer Society that intended to study the metastatic sites pattern of most common carcinomas, 984 patients with carcinoma of the colon were included, none of them with axillary lymph node metastasis. In this analysis that included a total of 4399 patients with primary carcinoma of the breast, oesophagus, lung, liver, stomach, pancreas, kidney, ovary, colon, rectum and prostate, $97 \%$ of the axillary lymph nodes metastasis came from the breast and $2 \%$ from the lung, with isolated cases of axillary invasion from primary carcinoma of oesophagus, liver and rectum [12].

In our literature review, we found four cases of late recurrence in axillary lymph nodes, always on the left side, in patients with primary colon carcinoma. In three of the cases, the primary tumor was of the descending colon and in the fourth case was of the cecum [1]-[4].

Metastasis to the axillary lymph nodes may occur either by haematogenous or lymphatic routes. There is no direct communication between colonic blood flow and axillary lymphatic system, since the colon blood flow reaches the liver first, through the portal vein. Additionally, most of colon lymphatic flow reaches the left venous angle through the thoracic duct and cisterna chyli. Since anatomic anomalies of the terminal portion of the thoracic duct have been described, the thoracic duct may communicate with the infra-clavicular lymphatic system in some patients. Therefore, colon cancer may spread to the axillary lymph nodes by lymphatic route through the thoracic duct and infra-clavicular lymphatic system [1]. Another possible dissemination pathway for axillary lymph node metastasis, is through lymphatic drainage channels that establish communication between the abdominal wall and the thoracic wall, through superficial abdominal lymphatics, namely the peri-umbilical ones, then to the parasternal lymph nodes and then to the internal mammary lymph nodes until they finally reach the axillary lymph nodes [3] [4]. This spread may also occur through deviations of lymphatic backflow from the para-aortic lymph nodes to the thoracic duct. These hypothesis seem to be the most likely spread ways, more than the hypothesis of haematogenous dissemination, though solid conclusions cannot be drawn [4].

In the presented case, a rare metastasis to a distant non-regional lymph node occurred, being the only metastasis diagnosed at disease presentation time, and sparing the most common metastasis sites (liver and lung). One hypothesis for this particular case is that the dissemination pathway occurred through haematogenous route, since the patient eventually presented with liver metastasis. However, these metastasis were not present at the time the axillary lymph node metastasis was detected, and so the lymphatic route dissemination seems to be the most likely one.

One important fact in this case is that the histological evaluation of the surgical specimen showed extracapsular invasion of the regional lymph nodes resected, which worsens the patient's prognosis. Besides that, this axillary lymph node is, by itself, a factor of bad prognosis, since it is a distant metastasis and, therefore, classifies this tumor as stage IV disease according to the TNM classification. These facts, along with the tumor perforation, explain why the patient came to develop so many distant metastasis (in the mesentery near the ileocolic anastomosis, in the abdominal lymph nodes, in the liver and in the abdominal fat) and died in such a short period of time.

\section{Conclusion}

Although the woman presented in this case report came to develop liver metastasis, the most likely spread path- 
way to the axillary lymph node seemed to be the lymphatic one. However, due to the rarity of this metastasis in tumors other than breast cancer, and given the little knowledge that we have of its spread pattern, it is impossible to draw conclusions.

\section{References}

[1] Suzuki, T., et al. (2006) Metastasis of Colon Cancer to Axillary Lymph Nodes. Jikeikai Medical Journal, 53, 167-170.

[2] Kikuchi, I., et al. (2012) A Case Report of Surgical Treatment for Axillary Lymph Node Metastasis from Descending Colon Cancer. GanTo Kagaku Ryoho, 39, 2252-2254.

[3] Gubitosi, A., et al. (2009) Unusual Metastasis of Left Colon Cancer: Considerations on Two Cases. Acta Biomedica, 80, 80-82.

[4] Chieco, P.A., et al. (2011) Solitary Left Axillary Metastasis after Curative Surgery for Right Colon Cancer. ANZ Journal of Surgery, 81, 846-847. http://dx.doi.org/10.1111/j.1445-2197.2011.05869.x

[5] Fujii, T., et al. (2011) Process of Distant Lymph Node Metastasis in Colorectal Carcinoma: Implication of Extracapsular Invasion of Lymph Node Metastasis. BMC Cancer, 11, 216. http://dx.doi.org/10.1186/1471-2407-11-216

[6] Yano, H., et al. (2006) Tumor Invasion of Lymph Node Capsules in Patients with Dukes C Colorectal Adenocarcinoma. Diseases of the Colon \& Rectum, 49, 1867-1877. http://dx.doi.org/10.1007/s10350-006-0733-9

[7] Tang, R., et al. (1995) Survival Impact of Lymph Node Metastasis in TNM Stage III Carcinoma of the Colon and Rectum. Journal of the American College of Surgeons, 180, 705-712.

[8] Gastrointestinal Tumor Study Group (1984) Adjuvant Therapy of Colon Cancer-Results of a Prospectively Randomized Trial. New England Journal of Medicine, 310, 737-743. http://dx.doi.org/10.1056/NEJM198403223101201

[9] Jass, J.R., Love, S.B. and Northover, J.M. (1987) A New Prognostic Classification of Rectal Cancer. Lancet, 1, 13031306. http://dx.doi.org/10.1016/S0140-6736(87)90552-6

[10] Pisanu, A., et al. (2011) Unusual Metachronous Isolated Inguinal Lymph Node Metastasis from Adenocarcinoma of the Sigmoid Colon. World Journal of Surgical Oncology, 9, 128. http://dx.doi.org/10.1186/1477-7819-9-128

[11] Llaguna, O.H., et al. (2010) Subcutaneous Metastatic Adenocarcinoma: An Unusual Presentation of Colon CancerCase Report and Literature Review. Case Reports in Oncology, 3, 386-390. http://dx.doi.org/10.1159/000321948

[12] Hess, K.R., et al. (2006) Metastatic Patterns in Adenocarcinoma. American Cancer Society, 106, 1624-1633. 
Scientific Research Publishing (SCIRP) is one of the largest Open Access journal publishers. It is currently publishing more than 200 open access, online, peer-reviewed journals covering a wide range of academic disciplines. SCIRP serves the worldwide academic communities and contributes to the progress and application of science with its publication.

Other selected journals from SCIRP are listed as below. Submit your manuscript to us via either submit@scirp.org or Online Submission Portal.
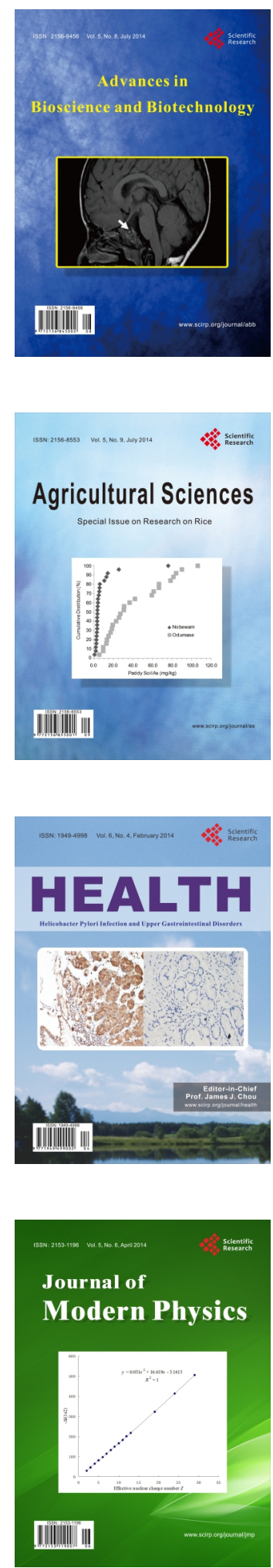
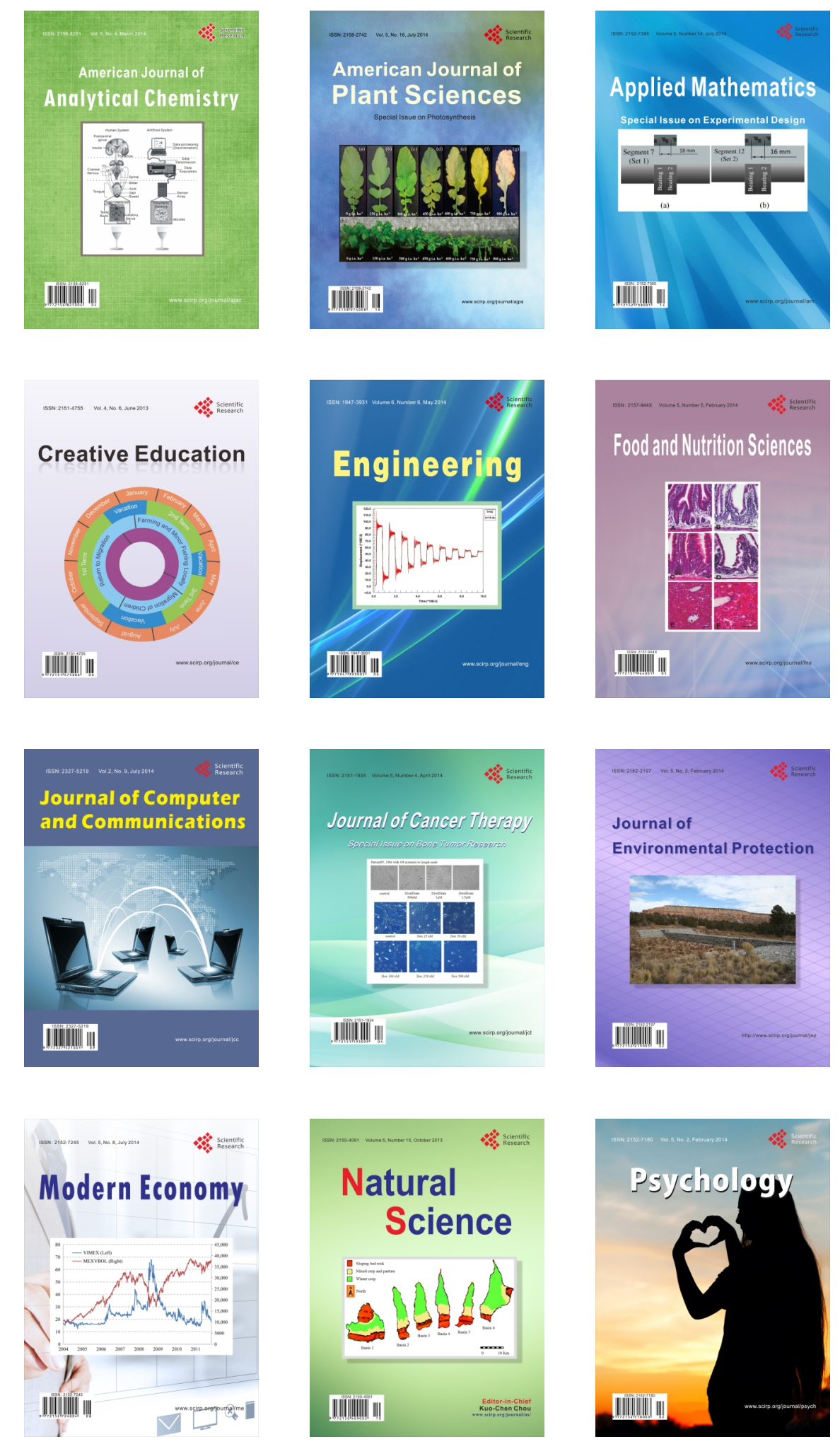